\title{
Improved Lossless Embedding and Extraction - A Data Hiding Mechanism
}

T. Venkat Narayana Rao ${ }^{1}$, Dr. A.Govardhan ${ }^{2}$ and Syed Jahangir Badashah ${ }^{3}$

\author{
${ }^{1}$ Professor, Tirumala Engineering College, Bogaram, Kesara, RR District, A.P, India \\ tvnrbobby@yahoo.com \\ ${ }^{2}$ Principal and Professor, Jawaharlal Nehru Technological University,Hyderabad, College \\ of Engineering, Jagityala, A.P, India, govardhan_cselyahoo.co.in \\ ${ }^{3}$ Research Scholar of Sathyabama University, Chennai, India, \\ syd_jahangireyahoo.co.in
}

\begin{abstract}
This paper proposes a reversible data hiding mechanism based on multiresolution analysis with difference expansion algorithm. Based on the multiresolution analysis of an image, gradient information of crude original image is exploited to select proper embedding areas. Adaptively embedding method based on Difference Expansion Algorithm is proposed to embed data into the host image without causing overflow / underflow and distortion problems. The original host image can be obtained upon the extraction of the embedded information. Driving application of reversible date hiding is authentic and can be used in some special applications, such as law enforcement, banks, medical and military fields, where original cover media is required for legal reason. Embedding data into cover media while keeping the media reversible opens a new door for linking some data with original media. With this proposed method we ensure that the Host information improves the compression efficiency, and thus the lossless data embedding capability is achieved. Experimental results signify that the proposed reversible data hiding method can embed a large payload with low visual distortion in the host image.
\end{abstract}

\section{KEYWORDS}

Reversible, Data hiding, Authentication, lossless extraction, Multi-resolution, Embedding, Peak Signal to Noise Ratio, stego-image. 


\section{INTRODUCTION}

Most multimedia data embedding techniques modify, and hence distort the host signal in order to insert the hidden or additional information. In many applications, the loss of host signal fidelity is affordable as long as original and modified signals are alike but permanent loss of signal fidelity is undesirable[1][2]. In most applications, the small distortion due to the data embedding is usually tolerable. The possibility of recovering the exact original host image is an enviable entity in many fields, like legal, medical and military imaging. Let us consider that sensitive documents (like bank cheques) when scanned, protected with an authentication scheme based on a reversible data hiding, and sent through the Internet super high way. In most cases, the watermarked documents will be sufficient to distinguish unambiguously the contents of the documents. However, if any uncertainty arises, the possibility of recovering the original unmarked document is very much important and desirable[15].

This gives rise to the need for Reversible (Lossless) Data Embedding techniques. These techniques, like their other lossy corresponding methods, inserting information bits by modifying the host signal, thus induce an embedding distortion.

Nevertheless, they also enable the removal of such distortions and the exact lossless restoration of the original host signal after extraction of embedded information[14]. Lossless data embedding techniques may be classified into one of the following two categories: Type I algorithms [2][3] employ additive spread spectrum techniques, where a spread spectrum signal corresponding to the information payload is superimposed on the host in the embedding phase. At the decoder, detection of the embedded information is followed by a restoration step where watermark signal is removed, i.e. subtracted, to restore the original host signal. Potential problems associated with the limited range of values in the digital representation of the host signal, e.g. overflows and underflows during addition and subtraction, are prevented by adopting modulo arithmetic. Payload extraction in Type-I algorithms is robust. On the other hand, modulo arithmetic may cause disturbing salt-and-pepper artifacts. In all Type-II algorithms, recovery of the original image is enabled by compressing, transmitting, and recovering these features. This property of the proposed method provides excellent compression of relatively simple image features. Earlier algorithms in the literature tend to select more complex features to improve the compression performance thus the lossless embedding [17] capacity.

Reversible data hiding has acknowledged more attention in recent years, where the invisible data (called the payload) is embedded into a host image to form a stego -image in a reversible fashion. In previous work [5], lossless compression techniques are applied to original image in order to create ability for embedding the payload. The payload (compressed data and secret data) is then embedded to form a stego-image. Fridrich [7][17] proposed a RS lossless embedding method, which classify the characteristics of image pixels into three types using a discrimination function. The payload is embedded using an invertible turn over operation according to how the characteristics of image pixels are classified. Vleeschouwer et al. [4] anticipated a reversible data embedding algorithm based on circular interpretation of transformations, where pixels of two pseudo-random sub-regions of a block are divided into two zones. Gray values in two zones are viewed as histograms. Slight rotations of these histograms in opposite directions allow for embedding a binary-value data. At the receiver, the embedded data and original image can be obtained according to the rotations of sub-regions. Thodi and Rodriguez [1][5][13] proposed a reversible data hiding technique based on prediction-error expansion and histogram shifting, in which a predictor is used to calculate the correlation between adjacent pixels; this enables the payload to be embedded with low image distortion. Tian [8] proposed a difference-expansion technique to reversibly embed a payload into digital images, in which the embedding data including the payload insertion map, and parts of the original least-significant bits are embedded into the original host image using a difference-expansion algorithm. These works aimed to achieve the desirous properties of high capacity and low distortion. However, the performances of 
these algorithms still can be improved. In this paper, we proposed a simple and efficient lossless data hiding scheme that improved on the data capacity without sacrificing visual quality. Based on a multi resolution analysis of an image, the gradient information of the coarse original in the Figure 1.1 and 1.2, the signal is denoted by the sequence $x[n]$, where $n$ is an integer. The low pass filter is denoted by $\mathrm{G}_{0}$ while the high pass filter is denoted by $\mathrm{H}_{0}$. At each level, the high pass filter produces detail information; $\mathrm{d}[\mathrm{n}]$, while the low pass filter associated with scaling function produces coarse approximations, $\mathrm{a}[\mathrm{n}]$.

At each decomposition level, the half band filters produce signals spanning only half the frequency band. This doubles the frequency resolution as the uncertainty in frequency is reduced by half. In accordance with Nyquist's rule if the original signal has a highest frequency of $\omega$, which requires a sampling frequency of $2 \omega$ radians, then it now has a highest frequency of $\omega / 2$ radians. It can now be sampled at a frequency of $\omega$ radians thus discarding half the samples with no loss of information. This decimation by 2 halves the time resolution as the entire signal is now represented by only half the number of samples. Thus, while the half band low pass filtering removes half of the frequencies and thus halves the resolution, the decimation by 2 doubles the scale.

With this approach, the time resolution becomes arbitrarily good at high frequencies, while the frequency resolution becomes arbitrarily good at low frequencies. The time-frequency plane is thus resolved as shown in figures below (i.e. Figure 1.1 and 1.2). The filtering and decimation process is continued until the desired level is reached. The maximum number of levels depends on the length of the signal. The DWT (Discrete Wavelet Transform) of the original signal is then obtained by concatenating all the coefficients, $a[n]$ and $d[n]$, starting from the last level of decomposition. As shown in the Figure 1.1 and 1.2 the reconstruction of the original signal from the wavelet coefficients. Basically, the reconstruction is the reverse process of decomposition. The approximation and detail coefficients at every level are up sampled by two, passed through the low pass and high pass synthesis filters and then added. This process is continued through the same number of levels as in the decomposition process to obtain and $\mathrm{H}_{\text {th }}$ original signal. The Mallet algorithm [12] works equally well if the analysis filters, $\mathrm{G}_{0}$ and $\mathrm{H}_{0}$, are exchanged with the synthesis filters $\mathrm{G}_{1}$

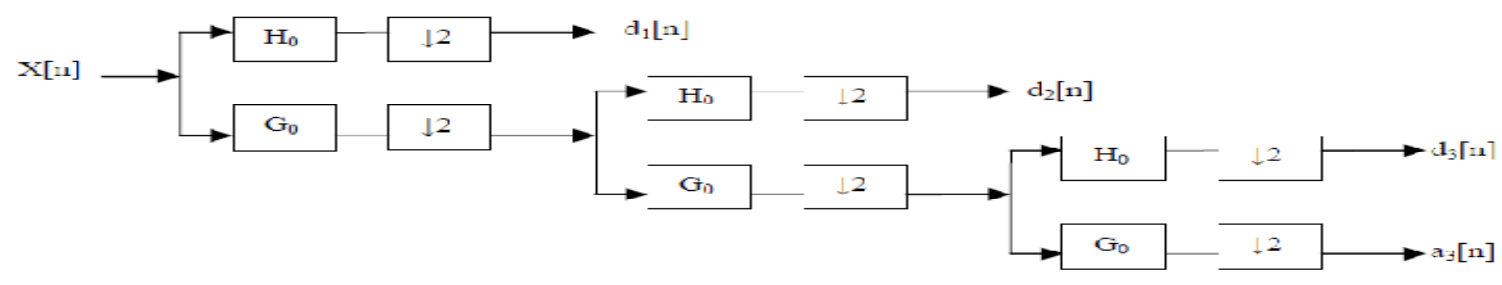

Figure 1.1Wavelet Decomposition

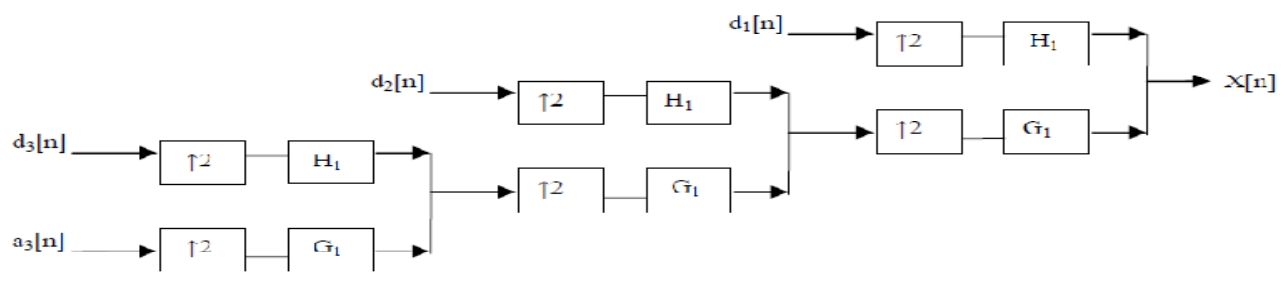

Figure 1.2 Wavelet Reconstruction 
The image in wavelet domain is used to select appropriate data embedding areas; and then the proposed adaptive embedding method based on Difference Expansion Algorithm can applied for a large payload with a little distortion.

\section{THE PROPOSED ALGORITHM}

The proposed lossless data hiding scheme is based on the multi resolution analysis and calculating the gradient image. Then an adaptively embedding method is proposed to support the concert of the projected scheme. Most data-hiding techniques alter and consequently distort the host signal in order to add the additional information. This distortion is usually minute but irreversible. Reversible data hiding insert information bits by altering the host signal, but enable the precise (lossless) restoration of the original host signal after extracting the embedded information. Sometimes, expressions like distortion-free, invertible, lossless or erasable watermarking are used as other names for reversible watermarking. In most applications, the small distortion due to the data embedding is usually acceptable. However, the possibility of recuperating the exact original image is a desirable property in many fields, like legal, medical and military imaging. Let us consider that sensitive documents (like bank checks) are scanned, protected with an authentication scheme based on a reversible data hiding, and sent through the Internet. In most cases, the watermarked documents will be adequate to distinguish clearly the contents of the documents. However, if any uncertainty arises, the possibility of recovering the original unhurt document is very difficult. To the best of our knowledge, none of the available reversible data hidings is adequate for watermarking binary images.

This technique provides high embedding capacities, allows complete recovery of the original host signal, and introduces only a small distortion between the host and image bearing the embedded data. The capacity of the scheme depends on the statistics of the host image. For typical images, the scheme offers ample capacity to address most applications. In applications requiring high capacities, the scheme can be modified to fine-tune the embedding level to meet up the capacity prerequisite. Figure 3. illustrate the hidden data in two popular images i.e. in a 512x512 format. Here we have narrated, the multi resolution analysis of an image is shown in Section 2.1. The details of the data embedding and extracting process are presented are described in Sections 2.2 and 2.3 respectively.

\subsection{Multi resolution Analysis}

The multi-resolution analysis based on the wavelet transform can decompose an image into a pyramidal arrangement for analyzing its characteristics [12][16].
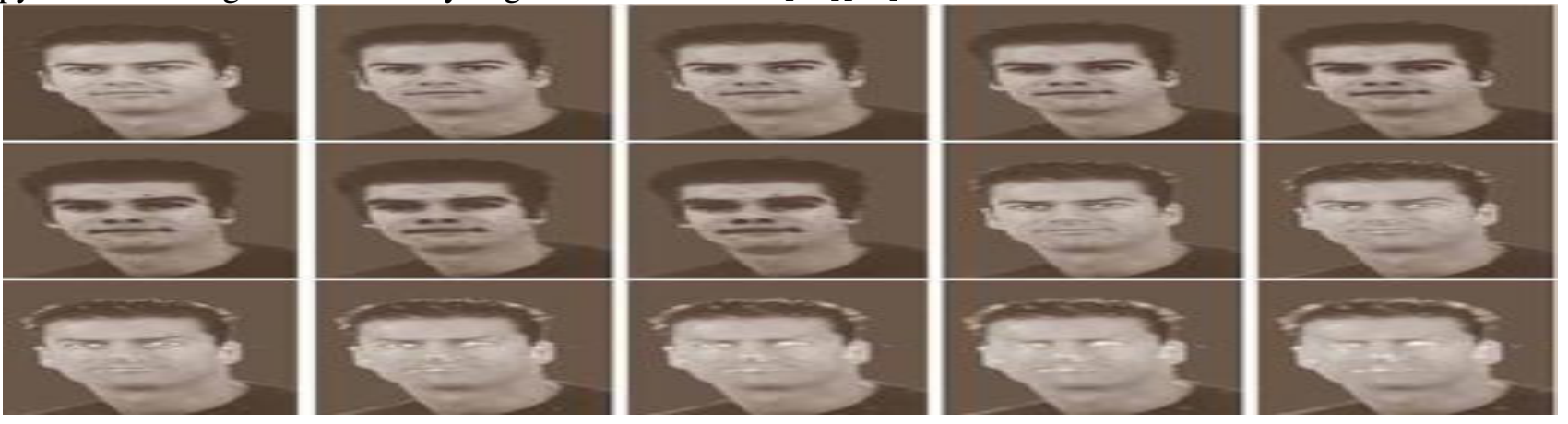

Figure 2. The concept data hiding and an example of the similar spectral content among detail sub images 
The 1-level multi-resolution analysis of an image results in coarse sub image and detail sub images. Furthermore, the spatial and frequency characteristics of an image can be shown concurrently. An example of multi-resolution analysis is shown in Fig. 2. It can be seen that the edge coefficients are larger than the other coefficients in the detail sub images.

Filters are one of the most widely used signal processing functions. Wavelets can be comprehended by iteration of filters with rescaling. The resolution of the signal, which is a metric of the amount of detail information in the signal, is determined by the filtering operations, and the scale is determined by up sampling and down sampling (sub sampling) operations as shown in Figure 1.1 and 1.2 .

The arrangement of edge coefficients in detail sub images are strong relative with those in coarse sub image. The notion of exploiting similar relationship among detail sub image has been used to promote the compression performance in many studies [10][18][11]. This is also a useful property for reversible data hiding. Hence, the proposed method is a lossless data hiding scheme. A lossless wavelet transform, Haar transform [2][3], can also be adopted for multi resolution analysis in the proposed algorithm.

\subsection{The Embedding Mechanism}

In the embedding process, the host image undergoes 1- level multi resolution analysis to produce coarse and detail sub images. Since the magnitudes of wavelet coefficients in the detail subimages are usually small, embedding data into these coefficients will result in less distortion on host image. Hence, the coefficients of detail sub images will be selected for embedding data. A payload $\{0,1\}$ is embedded into a selected wavelet coefficient using :$\mathrm{D}-\mathrm{emb}_{\mathrm{k}=} \mathrm{embed}\left(\mathrm{D}-\mathrm{emb} \mathrm{b}_{\mathrm{k}}, \mathrm{we}_{\mathrm{k}}\right)=2 \mathrm{D}-\mathrm{emb}_{\mathrm{k}}-\mathrm{we}_{\mathrm{k}}$

Where $\mathrm{D}-\mathrm{emb}_{\mathrm{k}}$ is the $k$ th selected wavelet coefficient and the $k, \mathrm{we}_{\mathrm{k}}$ is $k$ th element of the payload. Since the magnitudes of edge coefficients in the detail sub images are usually large, embedding any data into them will degrade the stego-image. To avoid embedding in the edge coefficients, edge detected in the coarse sub image to predict the edge coefficients in the detail sub images and the predicted edge coefficients of the detail sub images will be excluded from payload embedding. Thus, the embedding capacity and the visual quality of stego-image can be controlled by the edge predicting. The stricter the condition for edge detection, the fewer the edge coefficients, and thus more data embedding capacity and lower visual quality of stego-image. Besides, comparing to the earlier method [8] this is proposed to avoid wasting extra space to save payload insertion map and prevent the overflow and underflow problems. An estimating function $E$ is used to estimate the relationship between a wavelet coefficient and all possible values of a payload, with an outcome of either "safe" or "unsafe".

When a payload is embedded into the selected wavelet coefficient, the estimating function is applied to evaluate the coefficient. If four corresponding spatial pixels falls into a legal range or the estimated results of processed wavelet coefficients aren't affected, the estimated result is a safe state; otherwise, the estimated result is an unsafe state. According to the estimated result, the wavelet coefficient can be classified into three categories: prohibited, changeable, and expandable. If the estimated result of the wavelet coefficient is an unsafe state, this coefficient is categorized into the prohibited category; otherwise, this coefficient is categorized into the changeable category. The coefficients of changeable category can be further classified after a 1bit element of the payload is embedded. When a corresponding element of the payload is embedded into a changeable coefficient, the estimating function is applied to estimate the characteristic of the embedded coefficient. If the embedded coefficient is estimated as a safe state, the coefficient can be further classified into the expandable category.

After the selected wavelet coefficient is classified, the embedding strategy should vary with the category. For the expandable category, the 1-bit datum is directly embedded using Eq. (1). For the changeable category, and a flag F1 (whose value is 1) is embedded into the most succeeding and nonused changeable or expandable coefficient using Eq. (1). For the prohibited category, the 
value of the coefficient is maintained and a flag F0 (whose value is 0) is embedded into the most succeeding and nonused expandable or changeable coefficient using Eq. (1). Once all data are embedded, the inverse wavelet transform is applied to form a stego-image.

\subsection{The Extracting Mechanism}

The extracting process is the exact reversal of the embedding procedure. First, the 1-level multi resolution analysis is applied to the Stego-image to produce four sub images. Secondly, the edge detection result on the coarse sub image is used to predict the edge positions of detail sub images. Once the edge positions are detected, the payload insertion positions can be collected. Moreover, the estimating function is applied to classify the images as shown in Fig.(2) as an example of the similar spectral content among detail sub images characteristics of wavelet coefficients for extracting embedded data. According to the estimated results, each wavelet coefficient can be categorized roughly as either expandable or undesirable, according to whether the estimated result of the coefficient is a safe or an unsafe state, respectively. The embedded value stored in an expandable coefficient can be directly extracted using:$\mathrm{we}_{\mathrm{k}}=\operatorname{Extraction}\left(\mathrm{D}-\mathrm{emb} \mathrm{k}_{\mathrm{k}}\right)=\mathrm{D}-\mathrm{emb}_{\mathrm{k}} \bmod 2$

Where $\mathrm{D}-\mathrm{emb}_{\mathrm{k}}$ is the $k$ th selected wavelet coefficient in the stego-image. The category of undecidable coefficient can be further judged, according to the embedded value of the most succeeding and nonused expandable or undecidable coefficient. If the succeeding coefficient is an expandable coefficient and the embedded value is extracted, the embedded value can be viewed as a flag value. For embedded values of 1 and 0 , the undecidable coefficient is a changeable and prohibited coefficient, respectively, corresponding to flags F1 and F0. The embedded value stored in the changeable coefficient can be acquired using Eq. (2). If the succeeding coefficient is an undecidable coefficient, the characteristic of this undecidable coefficient is judged by the next most succeeding and nonused expandable or undecidable coefficient, iteratively. After all embedded data are extracted, the original wavelet coefficient of changeable and expandable coefficients coefficients can be obtained using:

$\mathrm{D}-\mathrm{emb}_{\mathrm{k}}=\left[\left(\mathrm{D}-\mathrm{emb} \mathrm{k}_{\mathrm{k}}+1\right) / 2\right]$

Finally, the 1-level inverse multi resolution analysis is applied to form a recovered image.

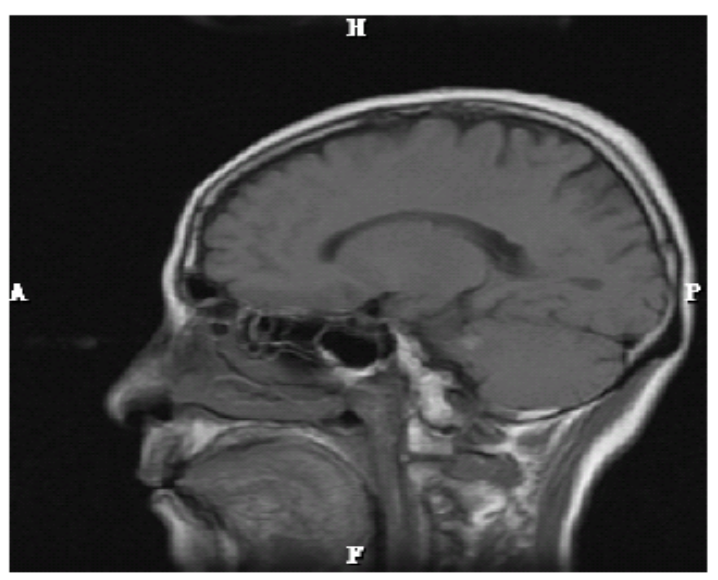

7000 bytes hidden in this medical image $(512 \times 512)$

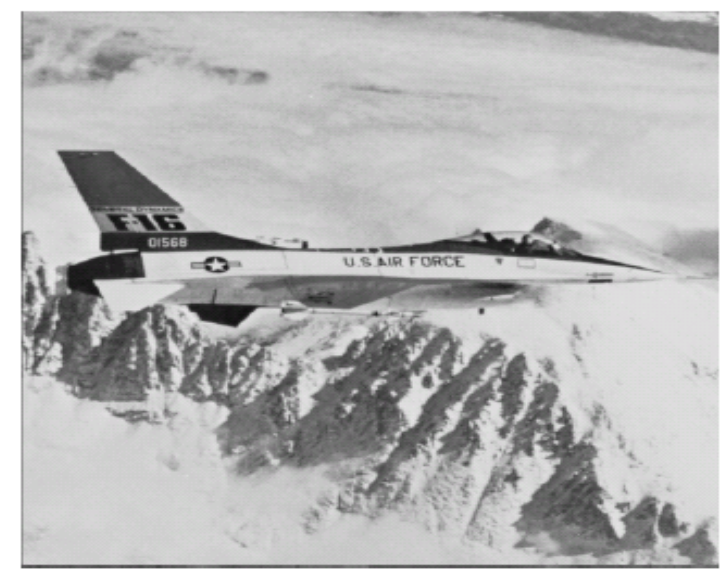

2000 bytes hidden in this image $(512 \times 512)$

Figure 3.Some sample images with hidden bytes but clueless and untraceable 


\section{EXPERIMENTAL RESULTS}

The grayscale standard images shown in Fig.(4) are used to evaluate the performance of the proposed algorithm [17][12]. All images are of $512 \times 512$ pixels size. The payload embedded was generated by randomly number generator. Sobel operator was also used for the edge detection . Among the previous best literatures [4][6][7] DE algorithm has greater performance in both payload limit and visual quality. In the first experiment, the performance of the proposed algorithm is compared with the DE algorithm [8]. Fig. (5a) and Fig.(5b) shows the capacity versus distortion comparison of the pepper image. Two observations can be made from the figures. First, the proposed algorithm can achieve more bits per pixel (bpp) embedding capacity than the DE algorithm same image quality: Secondly, the proposed algorithm has shown better image quality (better PSNR) than the DE algorithms at the same bpp embedding capacity. In the second experiment, the proposed embedding scheme is applied to various test images shown in Fig. (6a)nd Fig.(6b). The capacity versus distortion comparison of the test images is shown in Fig.(6a). except for the Mandrill image, the PSNRs of all stego-images are higher than $60 \mathrm{~dB}$ at
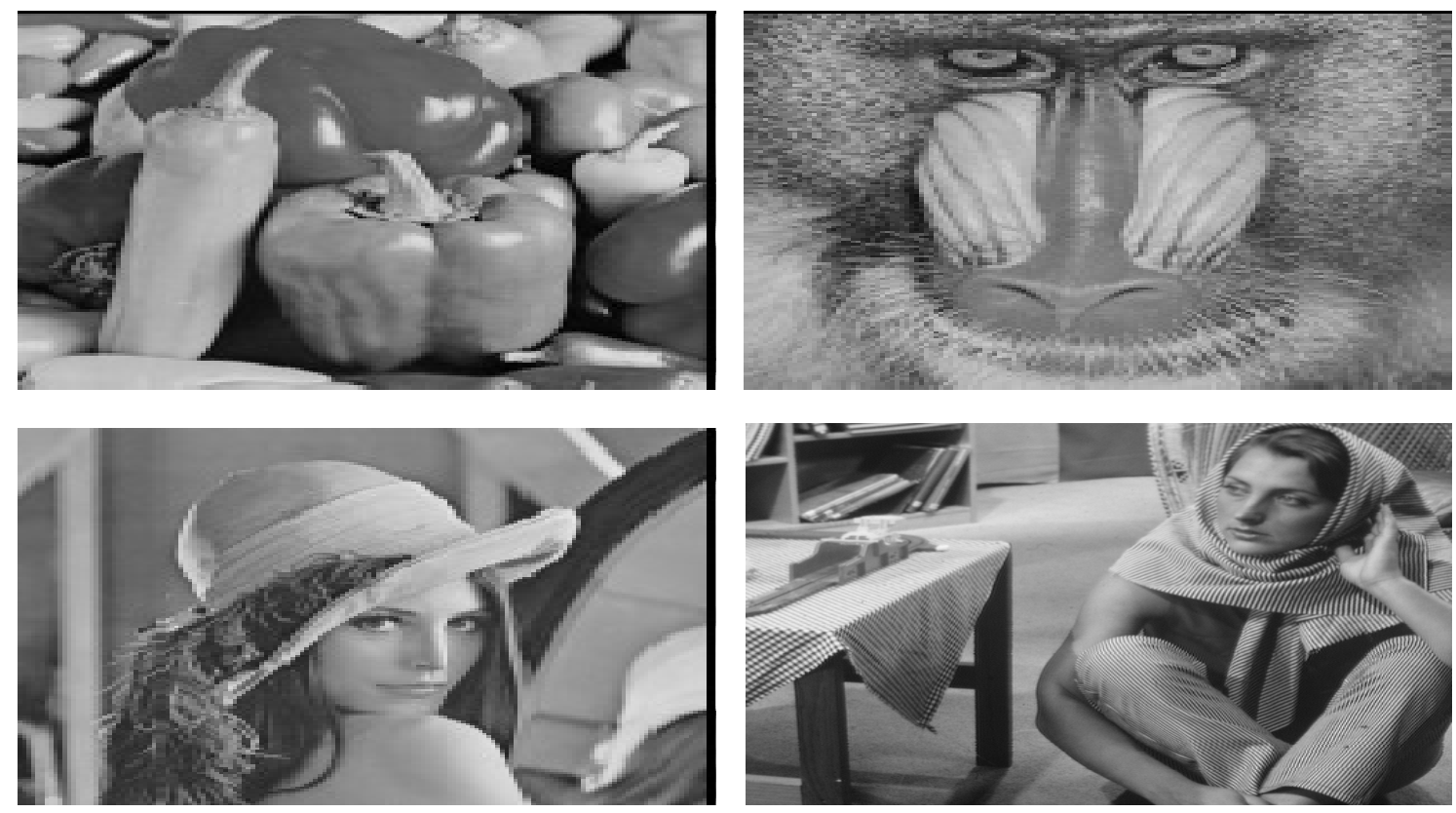

Figure 4. Grayscale test images subjected to data hiding.

0.05 bpp. The Mandrill image contains rich textures. Though the PSNR of the Mandrill stegoimage is lower than the others, there is no obvious visual flaw evident. 


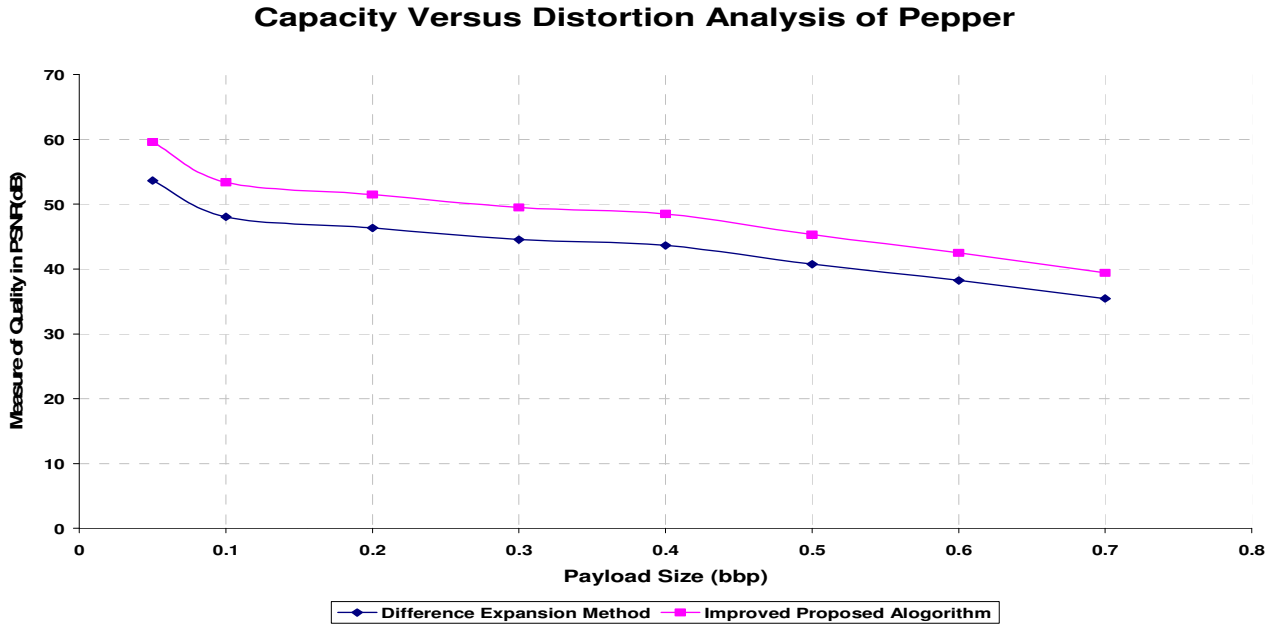

Figure 5a.Capacity Versus Distortion Analysis of Pepper.

Capacity Versus Distortion Anahyis of Pepper

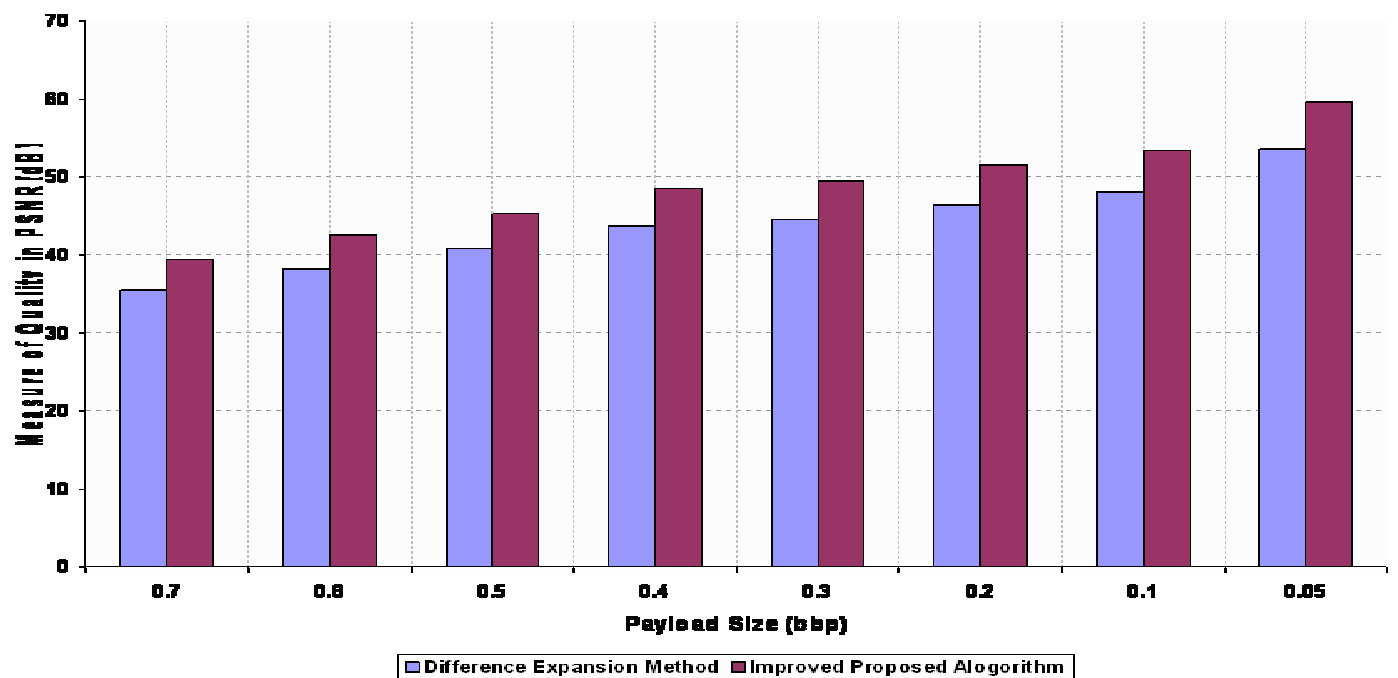

Figure 5b.Capacity Versus Distortion Analysis of pepper 


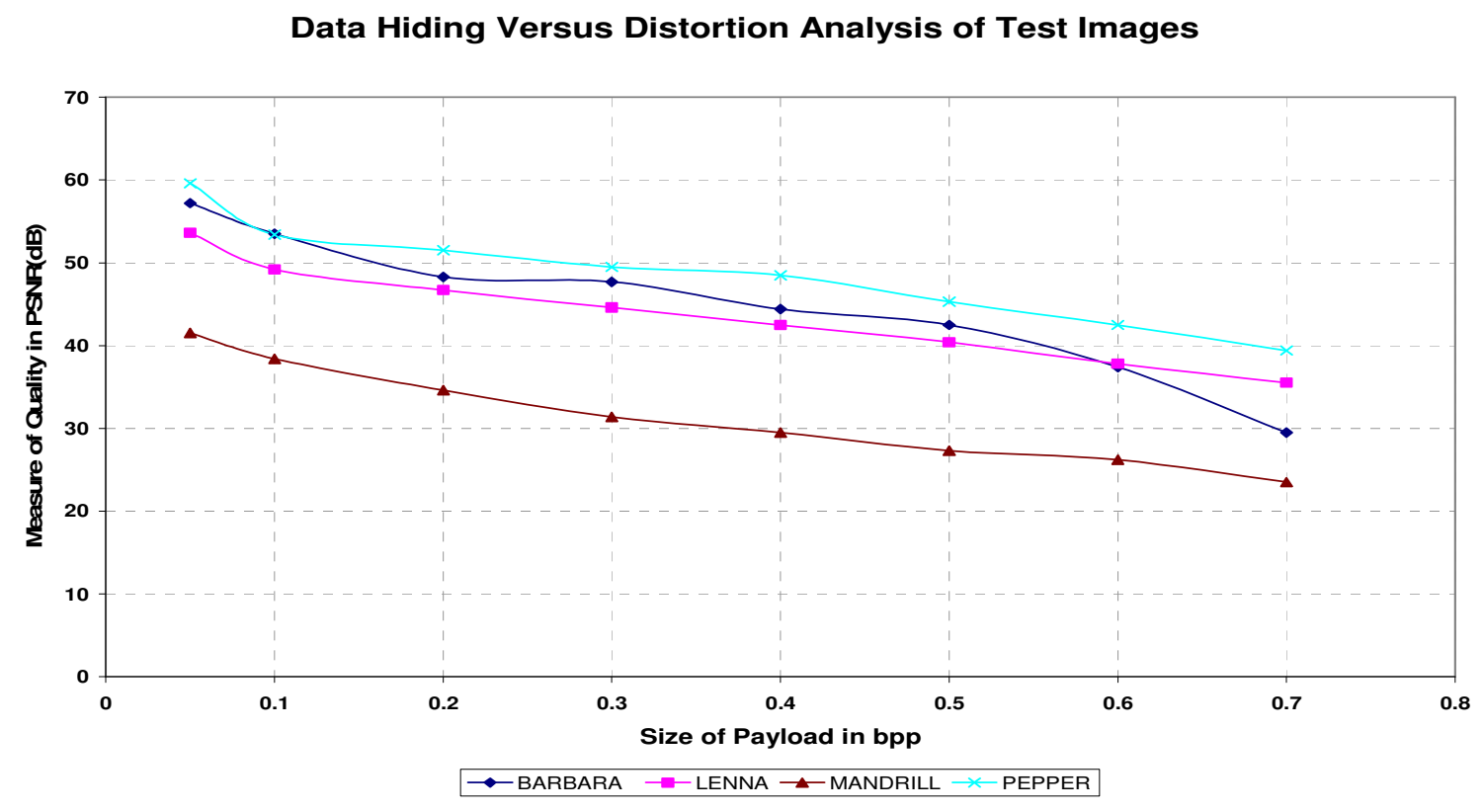

Figure 6a. Data Hiding Versus Distortion Analysis of Test Images

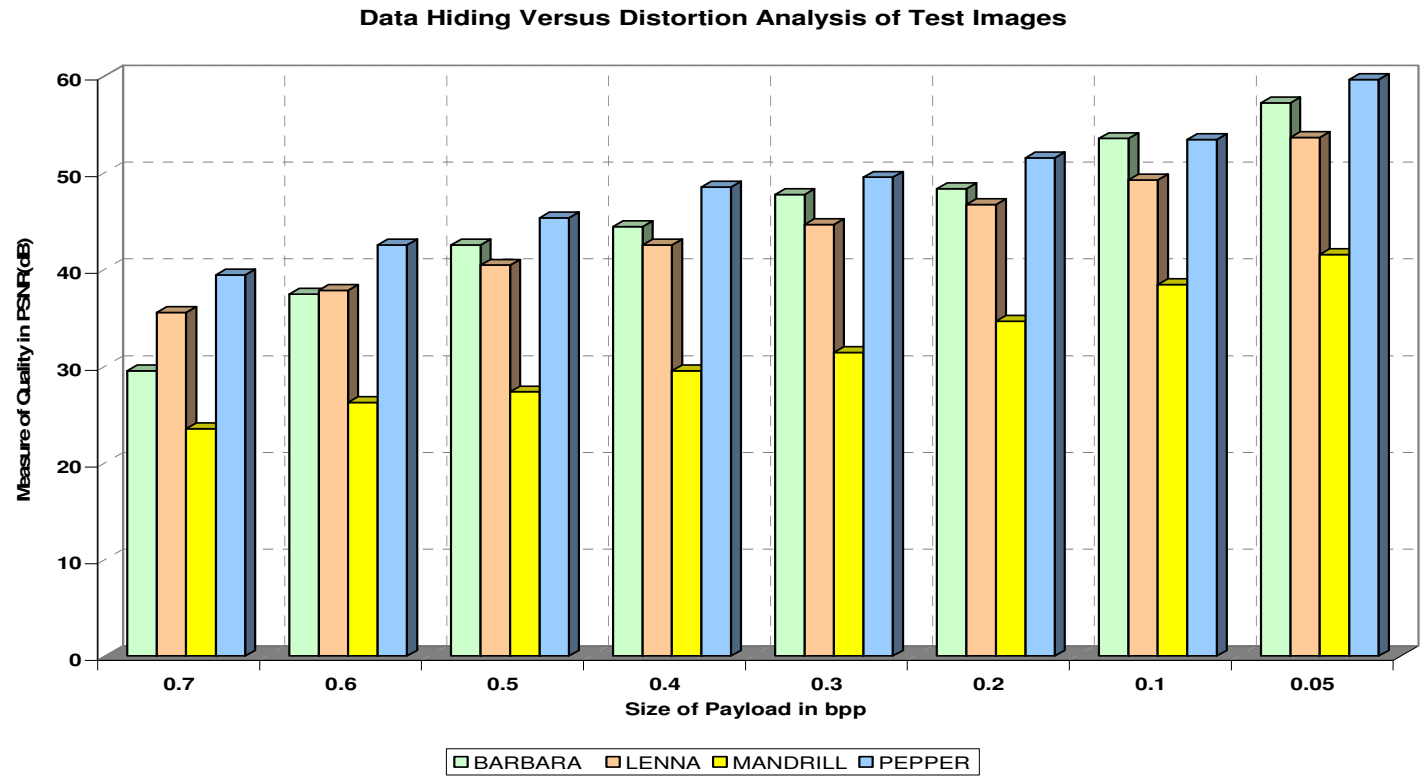

Figure 6b. Data Hiding Versus Distortion Analysis of Test Images 


\section{CONCLUSION}

A novel lossless (reversible) data embedding (hiding) technique is presented. The technique provides high embedding capacities, allows complete recovery of the original host signal, and introduces only a small distortion between the host and image bearing the embedded data. The capacity of the scheme depends on the statistics of the host image. For typical images, the scheme offers adequate capacity to address most applications. In applications requiring high capacity embedding, the scheme can be modified to adjust the embedding level to meet the capability requirements, thus trading off intermediate distortion for increased capacity. In such scenarios, the generalized embedding method proposed in the current paper is significantly advantaged over conventional embedding techniques because it offers finer granularity along the capacity distortion curve and its ability to fair well with higher PSNR values i.e near to $60 \mathrm{~dB}$ on large scale of images.

\section{REFERENCES}

[1] C.W. Honsinger, P.W. Jones, M. Rabbani, and J.C. Stoffel, "Lossless recovery of an original image containing embedded data,” US Pat. \#6,278,791, Aug 2001.

[2] Y. Q. Shi, "Reversible Data Hiding," Int. Workshop on Digital Watermarking 2004, (Seoul), Lecture Notes in Computer Science 3304, pp. 1-13, 2004

[3] A. R. Calderbank, I. Daubechies, W. Sweldens, and B.-L. Yeo, "Wavelet Transforms that Map Integers to Integers," Applied and Computational Harmonic Analysis, vol. 5, no. 3, pp. 332-369, 1998.

[4] C. D. Vleeschouwer, J. F. Delaigle, and B. Macq, "Circular Interpretation of Bijective Transformations in Lossless Watermarking for Media Asset Management,'IEEE Trans. Multimedia, vol. 5, no. 1, pp. 97-105, 2003.

[5] D. M. Thodi and J. J. Rodriguez, "Reversible Watermarking by Prediction-Error Expansion," in IEEE Symp. Image Analysis and Interpretation, pp. 21-25, 2004.

[6] G. Xuan, J. Zhu, J. Chen, Y. Q. Shi, Z. Ni, and W. Su, "Distortionless Data Hiding based on Integer Wavelet Transform,” Electronics Letters, vol. 38, no. 25, pp. 1646-1648, 2002.

[7] J. Fridrich, M. Goljan, and R. Du, "Lossless Data Embedding - New Paradigm in Digital Watermarking,” EURASIP J. Applied Signal Processing, vol. 2002, no. 2, pp. 185-196, 2002.

[8] J. Tian, "Reversible Data Embedding Using a Difference Expansion," IEEE Trans. Circuits and (a) (b)(c) (d) Fig. 2 Grayscale test images.Systems for Video Technology, vol. 13, no. 8, pp. 890-896, 2003.

[9] M. U. Celik, G. Sharma, E. Saber, and A. M. Tekalp,"Lossless Generalized-LSB Data Embedding," IEEETrans. Image Processing, vol. 14, no. 2, pp. 253-266, 2005.

[10] R. Rinaldo and G. Calvagno, "Image Coding by BlockPrediction of Multiresolution Subimages," IEEE Trans.Image Processing, vol. 4, no. 7, pp. 909-920, 1995.

[11] R. W. Buccigrossi and E. P. Simoncelli, "Image Compression via Joint Statistical Characterization in the Wavelet Domain,” IEEE Trans. Image Processing, vol. 8, no. 12, pp.1688-1701, 1999.

[12] S. G. Mallat, "A Theory for Multiresolution signal decomposition: The Wavelet Representation," IEEE Trans. Pattern Analysis and Machine Intelligence, vol.11, no.7, pp. 647-693, 1989.

[13] Ali Shariq Imran, M. Younus Javed, and Naveed Sarfraz Khattak “A Robust Method for Encrypted Data Hiding Technique Based on Neighborhood Pixels Information”,World Academy of Science, Engineering and Technology 312007.

[14] M. Goljan, J. Fridrich, and R. Du, "Distortion-free data embedding," Proceedings of 4th 
Information Hiding Workshop, pp. 27-41, Pittsburgh, PA, April, 2001.

[15] J. Fridrich, M. Goljan and R. Du, "Invertible authentication," Proc. SPIE, Security and Watermarking of Multimedia Contents, pp. 197-208, San Jose, CA, January-2001.

[16] Ruey-Ming Chao, Hsien-Chu Wu, Chih-Chiang Lee, and Yen-Ping Chu", A Novel Image Data Hiding Scheme with Diamond Encoding" EURASIP Journal on Information Security ,Volume 2009 , 9 pages, 2009.

[17] Dalian, Liaoning China "Data Hiding in Image Size Invariant Visual Cryptography",

"2008 3rd International Conference on Innovative Computing Information and Control", June 18-June 2008.

[18] Mahalingam Ramkumar and Ali N. Akansu, "Capacity Estimates for Data Hiding in Compressed Images“, IEEE TRANSACTIONS ON IMAGE PROCESSING, VOL. 10, NO. 8, AUGUST 2001.

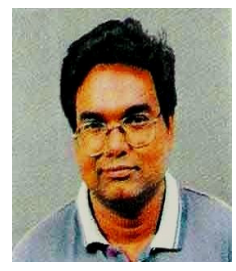

Author 1:T.Venkat Narayana Rao, received the B.E in Computer Technology and Engineering from Nagpur University, Nagpur, India , M.B.A (Systems) and M.Tech in computer Science from Jawaharlal Technological University, Hydarebad, A.P., India . He has 18 years of vast experience in Computer Engineering area pertaining to academics and industry related issues. He is presently Professor and Head, Department of Computer Science and Engineering, Tirumala Engineering College, Bogaram, Kesara , A.P , India. He is currently working on research area which include Digital Signal Processing, Digital Watermarking and Data Mining and other areas.

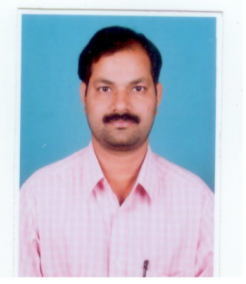

Author 2 : Dr.A.Govardhan did his BE in Computer Science and Engineering from Osmania University College of Engineering, Hyderabad, M.Tech from Jawaharlal Nehru University, Delhi and Ph.D from Jawaharlal Nehru Technological Univesity, Hyderabad. Presently he Principal and Professor of Computer Science and Engineering of JNTU, College of Engineering, Jagityala, A.P. He had worked as Professor and Head, Department of Computer Science and Engineering, JNT Univrsity Hyderabad. He has guided more than 100 M.Tech projects. He has 80 research publications at International/National Journals and Conferences. He is Member, Editorial Board of International Journal of Emerging Technologies and Applications in Engineering Technologies and Sciences ( IJ-ETA-ETS), and Editorial Board of International Journal of Computer Applications in Engineering, Technology and Sciences (IJ-CA-ETS). He has been a program committee member for various International and National conferences. He is also a reviewer of research papers of various conferences. He has delivered number of Keynote addresses and invited lectures. He is also a member in various professional bodies. His areas of interest include 
Databases, Data Warehousing \& Mining, Information Retrieval, Computer Networks, Image Processing and Object Oriented Technologies. He is presently a Professor and Head, Department of Computer Science and Engineering, JNTUH College of Engineering Hyderabad.

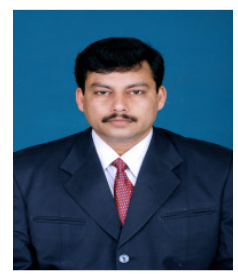

Author 3: Syed Jahangir Badashah received B.E.degree in Electronics \& Communication Engineering from Gulbarga University, in 2002, M.E.in Applied Electronics from Sathyabama University in 2005.He is currently doing research in image compression from Sathyabama University, He is having an experience of 8 years, in the field of teaching, presently working as Assistant Professor in the department of ECE, Madina Engineering College, Kadapa A.P, India. He is a member of IETE \&ISTE. E-mail:syd_jahangir@yahoo.co.in 